\title{
The Right Place at the Right Time: Medical Oncology Outpatients' Perceptions of Location of End-of-Life Care
}

\author{
Amy Waller, PhD a,b; Rob Sanson-Fisher, PhDa,b; Nicholas Zdenkowski, BMedc; Charles Douglas, PhD; \\ Alix Hall, PhD; ; and Justin Walsh, BAa,b
}

\begin{abstract}
Background: Helping people achieve their preferred location of care is an important indicator of quality end-of-life (EOL) care. Using a sample of Australian medical oncology outpatients, this study examined (1) their preferred location of EOL care; (2) their perceived benefits and worries of receiving care in that location; (3) the percentage who had discussed preferences with their doctor and/or support person; and (4) whether they wanted their doctor to ask them where they wanted to die. Methods: Adults with a confirmed diagnosis of cancer were approached between September 2015 and January 2016 in the waiting room of an Australian oncology outpatient clinic. Consenting participants completed a home-based pen-and-paper survey indicating preferred location of care, perceived benefits and worries of that location, whether they had discussed preferences with their doctors, and whether they were willing to be asked about their preferences. Results: A total of 203 patients returned the survey (47\% of those eligible). Less than half preferred to be cared for at home (47\%), $34 \%$ preferred a hospice/palliative care unit, and $19 \%$ preferred the hospital. Common benefits and worries associated with locations included perceived burden on others, familiarity of environment, availability of expert medical care, symptom management, and likelihood of having wishes respected. More patients had discussed preferences with their support persons (41\%) than doctors (7\%). Most wanted a doctor to ask them about preferred location of care (87\%) and thought it was important to die in the location of their choice (93\%). Conclusions: Patients were willing to have clinicians to ask them where they wanted to die, although few had discussed their preferences with doctors. Although home was the most preferred location for many patients, the overall variation suggests that clinicians should adopt a systematic approach to eliciting patient preferences.
\end{abstract}

Research into location of end-of-life (EOL) care offers insight into whether people are accessing resources that aim to improve quality of dying and support appropriate service planning. ${ }^{1}$ A recent population-level study of patients with cancer in 14 countries reported that between $12 \%$ and $57 \%$ of patients die at home and between $22 \%$

From a Health Behaviour Research Collaborative, School of Medicine and Public Health, The University of Newcastle, Callaghan; ${ }^{b}$ Hunter Medical Research Institute, New Lambton Heights; 'Department of Medical Oncology, Calvary Mater Newcastle, Waratah; ${ }^{d}$ Clinical Ethics and Health Law, The University of Newcastle, Callaghan; and ' Clinical Research Design and Statistics Support Unit, Hunter Medical Research Institute, New Lambton Heights, Australia.

Submitted May 14, 2017; accepted for publication August 9, 2017.

The authors have disclosed that they have no financial interests, arrangements, affiliations, or commercial interests with the manufacturers of any products discussed in this article or their competitors.

Dr. Waller is supported by an Australian Research Council DECRA and $78 \%$ die in the hospital. ${ }^{1}$ Where people are cared for at EOL is an important public health issue. EOL care delivered in hospitals is often resource-intensive, costly, and does not consistently meet patients' needs. Consequently, healthcare policies in many countries aim to reduce EOL hospital admissions and hospital mortality. ${ }^{2}$

(150101262). This research was supported by a National Health and Medical Research Council Partnership grant (1059760), a Strategic Research Partnership Grant (CSR 11-02) from Cancer Council NSW to the Newcastle Cancer Control Collaborative (New-3C), and infrastructure funding from the Hunter Medical Research Institute.

Author contributions: Study concept and design: Waller, Sanson-Fisher, Zdenkowski, Douglas. Patient recruitment: Zdenkowski, Douglas. Data acquisition: Waller, Walsh. Data analysis and interpretation: Hall. Manuscript preparation: All authors.

Correspondence: Amy Waller, PhD, Health Behaviour Research Collaborative, The University of Newcastle, Hunter Medical Research Institute, Callaghan NSW 2308, Australia. E-mail: amy.waller@newcastle.edu.au 
Waller et al

Despite the increasing availability of hospice facilities and palliative care (PC) services, significant gaps remain in their resources and utilization. Instead, home care has been advocated as a way of minimizing the demands placed on hospitals and long-term care facilities. ${ }^{4}$ However, home care may not be an appropriate or feasible option for everyone. Insufficient community-based services often shifts the burden of care to family members and friends. ${ }^{4}$ These individuals are not always adequately prepared to manage the complex needs of those nearing EOL at home, ${ }^{5}$ which can lead to multiple hospital admissions. ${ }^{6,7}$ Additionally, many patients do not have informal carers available to provide home care. ${ }^{8}$

Helping people achieve their preferred care location might be more indicative of high-quality EOL care. However, patients infrequently die in their preferred location. ${ }^{9}$ There is variation between studies in the proportion of people who prefer to be cared for at home $(25 \%-87 \%)$ or in a hospice $(9 \%-30 \%)$ at EOL. ${ }^{2}$ Despite hospitals being the most common place of death in many countries, few choose to be cared for in these facilities. ${ }^{2}$ Home care is also highly valued by informal carers $(25 \%-64 \%) .{ }^{10}$ Patients who are older (age $\geq 55 \mathrm{y}$ ), female, single, and of a higher socioeconomic status have been found to prefer hospice care, whereas those who are younger, male, of better physical health, and of poorer mental health have been found to prefer home care. ${ }^{11}$ However, few studies have explored how patients formulate preferences in order to choose one location over another. ${ }^{2}$ In one US study of terminally ill patients with cancer, perceived quality of life, the availability and capacity of family carers, the impact their choice would have on others, and quality of healthcare were major considerations in preference. ${ }^{12}$

Greater understanding of patient choices and the underlying reasons for these choices can help inform the development of more effective, patientcentered care models that may be more widely available and integrated across care settings. ${ }^{2}$ To do this, patients must be given opportunities to articulate and implement their choice through proper discussions with key individuals, including healthcare providers and support persons. When patients' preferences are known, their likelihood of dying in the location of their choice may be enhanced. Improved processes for the identification and communication of preferred place of death have been shown to in- crease the likelihood that patients achieve their wish to die at home (from $31 \%$ to $71 \%$ ). ${ }^{13,14}$ Those who did not express a preference were 3 times more likely to die in the hospital. ${ }^{13,14}$ The extent to which patients want to discuss, and have already discussed, their preferences for location of care with providers and support persons must first be established.

Using a sample of Australian medical oncology outpatients, this study examined (1) their preferred location of EOL care and the importance of achieving this preference; (2) their perceived benefits and worries of receiving care in that location; (3) the percentage who had discussed preferences for EOL care with their doctor and/or support person; and (4) their willingness to be asked by their doctor where they wanted to die.

\section{Methods}

\section{Design}

A cross-sectional survey was performed of medical oncology outpatients recruited from a single tertiary treatment center. Data on disease stage were extracted by a medical oncologist from patient medical records.

\section{Participants}

Eligible patients had a confirmed cancer diagnosis, were attending the clinic for their second or subsequent appointment (to ensure that patients had experienced cancer care), were aged $\geq 18$ years, were able to read and understand English, and were deemed by clinical staff to be physically and mentally able to give informed consent and to complete the survey.

\section{Procedure}

Clinic staff identified eligible patients from daily clinic lists. A trained research assistant obtained informed consent by consecutively approaching eligible patients while they waited for their appointment. Given the potentially sensitive nature of the items, oncologists asked that consenting patients complete a pen-and-paper survey at home. Nonresponders received a reminder letter 2 weeks later.

\section{Outcome Measures}

Preferred Location of Care: Participants were asked, "If you had a choice, where would you prefer to be 
cared for at the end of life?" Response options were: (1) in your own home, (2) in a relative's home, (3) in a hospital, or (4) in a hospice/PC unit (ie, inpatient hospice facility or PC unit).

Perceived Benefits and Worries: Participants then answered a list of potential benefits and worries specific to their preferred place of care, including home, hospital, or hospice/PC. Participants were asked, "Which of the following might be benefits to receiving care at the end of life in [preferred location]?" Participants were asked to rank, in order of preference, the top 3 most important benefits from the list provided (ie, "1" represented the most important perceived benefit). Participants were then asked, "Which of the following might you be most worried about if you were receiving care at the end of life in [preferred location]?" and rank their top 3 most important worries in order of their preference.

Discussion of Preferences With Others: Participants were asked to respond either "yes" or "no" to the question, "Have you ever discussed where you would like to be cared for at the end of life with your (a) doctor and/or (b) support person?"

Willingness to Discuss Preferences: Under a heading that specified, "What you would want if you were facing the end of your life," participants were also asked to respond to the statement "I would want my healthcare team to ask me... where I would prefer to die," with options ranging from strongly disagree to strongly agree.

Dying in Location of Choice: Under a heading that specified, "What you would want if you were facing the end of your life," participants were also asked to respond to the statement, "I would want to be able to die in the location of my choice (eg, at home, hospital, hospice, other)," with options ranging from strongly disagree to strongly agree.

\section{Sociodemographic and Clinical Characteristics:} Sociodemographic and clinical characteristics included self-reported sex, age, postcode, and cancer type. Patient estimation of life expectancy was obtained via an item "What is your estimation of your life expectancy?" Response options included "less than 6 months," "6 months to 1 year," "1 to 2 years," "more than 2 years," "don't know but I would like this information," and "don't know and
I do not want this information." Potential for cure was a dichotomous variable ("potentially curable versus incurable"), obtained using data extracted from the patients' medical records and reviewed by a medical oncologist.

Statistical Analysis: Stata/IC 11.1 (StataCorp LP, College Station, TX) was used for all analyses. Consent bias for sex was assessed with chi-square analyses. Frequency and percentage data were used to describe (1) patients' preferred place to receive EOL care and whether they agreed or disagreed it was important to die in the location of their choice; (2) whether patients had discussed their preference with their doctor and/or support person; and (3) whether patients would like to be asked by their healthcare team where they would like to die. The percentage and frequency of participants ranking each "benefit" and "worry" as either 1, 2, or 3 was calculated. To assess the relevant importance that patients attributed to each of the benefits and worries, the rank of each item was reverse scored and then the sum for each item was calculated (eg, each time an item was ranked 1 , it received a score of 3; items ranked 2 received a score of 2; and items ranked 3 received a score of 1 ).

\section{Ethics Approvals}

The University of Newcastle Human Research Ethics Committee (H-2014-0411) and the ethics committee of the participating health service approved the study $(14 / 11 / 19 / 4.04)$.

\section{Results}

\section{Sample}

Of the 436 eligible patients approached, 366 consented to participate ( $83 \%$ of eligible), and 203 ( $47 \%$ of eligible) returned a survey. Table 1 presents the characteristics of patients who returned a survey.

\section{Preferred Location for EOL Care}

Overall, $47 \%$ of patients $(n=93)$ indicated a preference to be cared for either in their own home or in the home of a relative. Just more than one-third of patients $(n=66 ; 34 \%)$ indicated a preference to be cared for in a hospice or PC unit, whereas 19\% $(n=37)$ indicated they would prefer to be cared for in a hospital. 
Waller et al

\begin{tabular}{|c|c|}
\hline Characteristics & $\begin{array}{c}\text { Sample } \\
\mathrm{n}(\%)\end{array}$ \\
\hline \multicolumn{2}{|l|}{ Sex } \\
\hline Male & $84(41 \%)$ \\
\hline Female & $119(59 \%)$ \\
\hline \multicolumn{2}{|l|}{ Age, y } \\
\hline Mean (SD) & 65 (11.5) \\
\hline \multicolumn{2}{|l|}{ Australian born } \\
\hline Yes & $173(85 \%)$ \\
\hline No & $25(12 \%)$ \\
\hline Missing & $5(2.5 \%)$ \\
\hline \multicolumn{2}{|l|}{ Cancer type } \\
\hline Breast & $69(34 \%)$ \\
\hline Colorectal & $35(17 \%)$ \\
\hline Prostate & $21(10 \%)$ \\
\hline Lung & $21(10 \%)$ \\
\hline Other & $55(27 \%)$ \\
\hline Missing & $2(1 \%)$ \\
\hline \multicolumn{2}{|c|}{ Current cancer status (from medical records) } \\
\hline Curable & $98(48 \%)$ \\
\hline Incurable & $94(46 \%)$ \\
\hline Missing & $11(5.4 \%)$ \\
\hline \multicolumn{2}{|l|}{ Life expectancy } \\
\hline$\leq 2 \mathrm{y}$ & $21(10 \%)$ \\
\hline$>2 \mathrm{y}$ & 69 (34\%) \\
\hline Unsure & $108(53 \%)$ \\
\hline Missing & $5(2.5 \%)$ \\
\hline
\end{tabular}

\section{Importance of Achieving Preference}

Most patients ( $n=185 ; 93 \%)$ strongly agreed/agreed that it was important for them to be able to die in the location of their choice.

\section{Patient Perceptions of Most Important Benefits and Worries}

Home Care: The top 5 perceived benefits of home care were, in descending order: receiving care from family and friends, being in a familiar environment, being physically close to their loved ones, not being alone, and their family being able to have a more "normal life." The biggest worries were, in descending order: not having pain managed well, concern about emotional "scarring" of family/friends, not having access to expert medical care, family/friends not knowing what to do during/after death, and not being found for several hours after death. See supple- mental eAppendix 1 (available with this article at INCCN.org).

Hospice: The top 5 perceived benefits of hospice care were, in descending order: pain being managed well, not being a burden to family and friends, family being able to have a more "normal life," access to specialized medical care, and having medical staff on call. The top 5 perceived worries were, in descending order: being in an unfriendly and clinical environment, having family's/friends' last memory of them being in a hospice facility, lack of privacy, not being ready to die, and being isolated. See supplemental eAppendix 2.

Hospital: The top 5 perceived benefits were, in descending order: pain being managed well, not being a burden to family and friends, having medical staff on call, family being able to have a more "normal life," and having access to lots of medical care. The top 5 worries were, in descending order: not having their wishes respected, not being treated respectfully, having family's/friends' last memory of them being in a hospital, getting an infection or becoming sicker, and hospital being a distance for visitors to travel. See supplemental eAppendix 3.

\section{Discussions About Preferred Location of Care}

Overall, fewer than half of all patients $(n=82 ; 41 \%)$ reported having discussed where they wanted to be cared for at EOL with either their doctor or support person. More patients had discussed it with their support person $(41 \% ; n=79)$ than with their doctor $(6.7 \% ; n=12)$.

\section{Desire to be Asked About Preferred Location of EOL Care}

Most patients indicated they would want their healthcare provider to ask them about where they would prefer to die if facing EOL $(n=176 ; 86.7 \%)$.

\section{Discussion}

Almost all patients emphasized that being able to die in the location of their choice was important to them. Nearly half of the patients surveyed indicated a preference for receiving care at home, which is consistent with findings of previous studies. ${ }^{10}$ Onethird of patients indicated a preference for receiving care in a hospice/palliative unit. This proportion is 
higher than reported in studies conducted a decade ago, ${ }^{11,15}$ which may reflect a growing recognition of the availability and potential benefits of these services among patients with cancer, ${ }^{16,17}$ and be a result of increasing efforts to integrate oncology and PC services. ${ }^{18}$ Although more than half of Australians die in hospitals, only one-fifth of patients selected the hospital as their most preferred location of care. The perception that dying in an institution is a failure of the system does not acknowledge the potential cost to those called on to care for the person at home. ${ }^{19}$ Patients dying at home may have unintended consequences for caregivers, such as deterioration in physical health, inability to return to work, and protracted grief. ${ }^{2,19,20}$ Further research is needed to establish whether the involvement of PC shifts care from institutions to home, or whether dying at home is better than dying in a hospital.,19 The limited evidence for benefits of one location over another, combined with the substantial proportion of patients who prefer to die in settings outside the home, suggests that although investment in services such as home PC may help reduce the burden on hospitals and inpatient hospices, improvements in EOL care should continue across all care settings.

When asked, patients in this study identified interpersonal factors, such as the opportunity to be cared for by family or friends and being in a familiar environment, as important benefits of receiving care in the home. In contrast, patients reported being worried about the quality of medical care available to them, as well as the potential burden on their family/ friends. These concerns have also been reported by other patient groups. ${ }^{12,21}$ Although there is conflicting evidence about the quality of care received in inpatient settings versus at home, ${ }^{2}$ some patients perceive that having access to professional medical care in inpatient settings is essential. People may also feel a sense of security from receiving care at a hospital with which they are already familiar.

The perception that higher-quality EOL care is delivered in settings outside the home was also echoed in the views of those indicating a preference for care in institutions. Patients identified availability of expert medical care and pain management, as well as reduced burden on family/friends, as important. However, participants were worried about being in an unfriendly environment that lacked privacy, not receiving care in accordance with their wishes, and the potential emotional impact on family/friends of having their last memories of the patient be in a facility. Some of these concerns can be easily addressed, such as by allowing the patient, family, and friends to see the facility and meet some of the staff to address concerns about the clinical environment. Other concerns, such as worry about not receiving care in accordance with wishes, highlight the need for improved communication between patients, families, and providers regarding goals of care, and for sharing plans with all those involved. ${ }^{22} \mathrm{Al}$ though less frequently endorsed, practical concerns such as family/friends not knowing what to do after death could be addressed through education. Ensuring the accuracy of patients' perceptions of the potential costs and benefits of different EOL care locations is critical; inaccurate perceptions may result in unwanted medical care and adversely impact patient and family outcomes. ${ }^{23,24}$

Almost half of the patients surveyed had spoken to their support person about their preference. However, very few patients had discussed their preferences with their doctor. This is surprising because most indicated that they would want their doctor to ask them where they wanted to die if facing EOL. Further, almost half of the sample had incurable cancer. The low rates may reflect barriers previously identified in the literature, such as clinicians' being time-poor or having concerns about their ability to conduct discussions well, or their perception that patients do not want to discuss these topics. ${ }^{25-27}$ Conversely, patients and support persons may not be asking about this issue, because they may perceive that the doctor does not have time or that the doctor does not feel comfortable discussing the topic. ${ }^{27}$

Strategies to enhance the knowledge and skills of both patients and clinicians in effectively initiating and responding to discussions are needed. Question prompt lists may improve patient and family knowledge and comfort in raising concerns. Improvements in patient-provider communication about EOL and prognostic issues have been reported when such tools are implemented..$^{28,29}$ For clinicians, hypothetical scenarios may be an effective means of introducing EOL issues. For instance, clinicians may ask, "The future is uncertain for us all. If you were facing EOL care, where would you like this to occur: at home, hospice, or hospital? Would you like me to give you some ideas about care in each of these set- 
Waller et al

tings and see what we can do to increase the probability you get what you want?" Given the key role of family/friends in informal care and the influence that perceived family burden may have on patient choices, it would be preferable for support persons to be involved in these discussions to ensure that the patient's choice can be supported.

\section{Strengths and Limitations}

This is one of few studies that has quantitatively examined perceptions of the potential benefits and worries associated with preferred location of EOL care among a large group of medical oncology outpatients. There are a number of limitations to this study. First, we acknowledge the low response rates for the surveys, which may be due to patients taking surveys home to complete and not returning them or to the nature of the topic. The cross-sectional nature of the data is also a limitation, given the potentially dynamic nature of decision-making about preferences for place of death and the influences of deterioration in physical functioning, diminished family resources, or available healthcare on these preferences. A qualitative component exploring patientperceived worries and benefits may have provided a more in-depth understanding of preferences for loca- tion of EOL care. It cannot be assumed that preferences for location of care will remain stable over time; how often and why preferences change must be better understood. Prospective longitudinal data examining patient preferences, the views of their caregivers, and the extent to which individual preferences are met are critical to advance the field.

\section{Conclusions}

Dying in the location of their choice is important to patients. Although patients were willing to have healthcare providers ask them where they wished to die, few discussed it with their doctor. Given the overall variation in preferred location of EOL care among patients, healthcare providers should take a systematic approach to eliciting patient preferences. This can help ensure that reasons for patients' choices are expressed and understood, and that patients have the appropriate support to achieve their preferences.

\section{Acknowledgments}

The authors would like to acknowledge research support from Lucy Boyd, Judy Hollingworth, and Natalie Dodd. They also wish to thank hospital staff, patients, and families for their contribution to this research.

\section{References}

1. Higginson IJ, Sarmento VP, Calanzani N, et al. Dying at home-is it better: a narrative appraisal of the state of the science. Palliat Med 2013;27:918-924.

2. Cohen J, Pivodic L, Miccinesi G, et al. International study of the place of death of people with cancer: a population-level comparison of 14 countries across 4 continents using death certificate data. Br J Cancer 2015;113:13971404.

3. Meier D, McCormack E, Lagman RL. Hospice: philosophy of care and appropriate utilization in the United States. UpToDate. Available at https:// www.uptodate.com/contents/hospice-philosophy-of-care-and-appropriateutilization-in-the-united-states. Updated February 3, 2017. Accessed December 13, 2017.

4. Swerisson H, Duckett S. Dying well. Available at: https://grattan.edu.au/wpcontent/uploads/2014/09/815-dying-well.pdf. Grattan Institute Report No 2014-10, September 2014. Accessed December 13, 2017.

5. Gallagher R, Krawczyk M. Family members' perceptions of end-of-life care across diverse locations of care. BMC Palliat Care 2013;12:25.

6. Goldsbury DE, O'Connell DL, Girgis A, et al. Acute hospital-based services used by adults during the last year of life in New South Wales, Australia: a population-based retrospective cohort study. BMC Health Serv Res 2015;15:537.

7. O'Connell DL, Goldsbury DE, Davidson P, et al. Acute hospital-based services utilisation during the last year of life in New South Wales, Australia: methods for a population-based study. BMJ Open 2014;4:e004455.

8. Aoun SM, Breen LJ, Howting D. The support needs of terminally ill people living alone at home: a narrative review. Health Psychol Behav Med 2014;2:951-969.

9. Billingham MJ, Billingham SJ. Congruence between preferred and actual place of death according to the presence of malignant or non-malignant disease: a systematic review and meta-analysis. BMJ Support Palliat Care 2013;3:144-154.

10. De Roo ML, Miccinesi G, Onwuteaka-Philipsen BD, et al. Actual and preferred place of death of home-dwelling patients in four European countries: making sense of quality indicators. PLoS One 2014;9:e93762.

11. Gomes B, Calanzani N, Gysels $M$, et al. Heterogeneity and changes in preferences for dying at home: a systematic review. BMC Palliat Care 2013;12:1-13.

12. Foreman LM, Hunt RW, Luke CG, et al. Factors predictive of preferred place of death in the general population of South Australia. Palliat Med 2006;20:447-453.

13. Tang ST. When death is imminent: where terminally ill patients with cancer prefer to die and why. Cancer Nurs 2003;26:245-251.

14. Arnold E, Finucane AM, Oxenham D. Preferred place of death for patients referred to a specialist palliative care service. BMJ Support Palliat Care 2015;5:294-296.

15. Oxenham D, Finucane A, Arnold E, Russell P. Delivering preference for place of death in a specialist palliative care setting. BMJ Qual Improv Rep 2013;2:pii: u201375.w897.

16. Agar M, Currow DC, Shelby-James TM, et al. Preference for place of care and place of death in palliative care: are these different questions? Palliat Med 2008;22:787-795.

17. Dionne-Odom JN, Azuero A, Lyons KD, et al. Benefits of early versus delayed palliative care to informal family caregivers of patients with advanced cancer: outcomes from the ENABLE III randomized controlled trial. J Clin Oncol 2015;33:1446-1452.

18. Temel JS, Greer JA, Muzikansky A, et al. Early palliative care for patients with metastatic non-small-cell lung cancer. N Engl J Med 2010;363:733742 .

19. Hui $\mathrm{D}$, Bruera $\mathrm{E}$. Integrating palliative care into the trajectory of cancer care. Nat Rev Clin Oncol 2016;13:159-171.

20. Currow DC, Agar M, Abernethy AP. Hospital can be the actively chosen place for death. J Clin Oncol 2013;31:651-652. 
Patient Perceptions of EOL Care Location

21. Abernethy AP, Currow DC, Fazekas BS, et al. Specialized palliative care services are associated with improved short- and long-term caregiver outcomes. Support Care Cancer 2008;16:585-597.

22. Fried TR, van Doorn C, O'Leary JR, et al. Older persons' preferences for site of terminal care. Ann Intern Med 1999;131:109-112.

23. Australian Commission on Safety and Quality in Health Care. National consensus statement: essential elements for safe and high-quality end-oflife care. Available at: https://www.safetyandquality.gov.au/wp-content/ uploads/2015/05/National-Consensus-Statement-Essential-Elementsforsafe-high-quality-end-of-life-care.pdf. Accessed December 13, 2017.

24. Wright AA, Zhang B, Keating NL, et al. Associations between palliative chemotherapy and adult cancer patients' end of life care and place of death: prospective cohort study. BMJ 2014;348:g1219.
25. Weeks JC, Catalano PJ, Cronin A, et al. Patients' expectations about effects of chemotherapy for advanced cancer. N Engl J Med 2012;367:1616-1625.

26. Keating NL, Landrum MB, Rogers SO, et al. Physician factors associated with discussions about end-of-life care. Cancer 2010;116:998-1006.

27. You JJ, Downar J, Fowler RA, et al. Barriers to goals of care discussions with seriously ill hospitalized patients and their families: a multicenter survey of clinicians. JAMA Intern Med 2015;175:549-556.

28. Larson DG, Tobin DR. End-of-life conversations: evolving practice and theory. JAMA 2000;284:1573-1578.

29. Dimoska A, Butow PN, Lynch J, et al. Implementing patient questionprompt lists into routine cancer care. Patient Educ Couns 2012;86:252-258.

See JNCCN.org for supplemental online content.

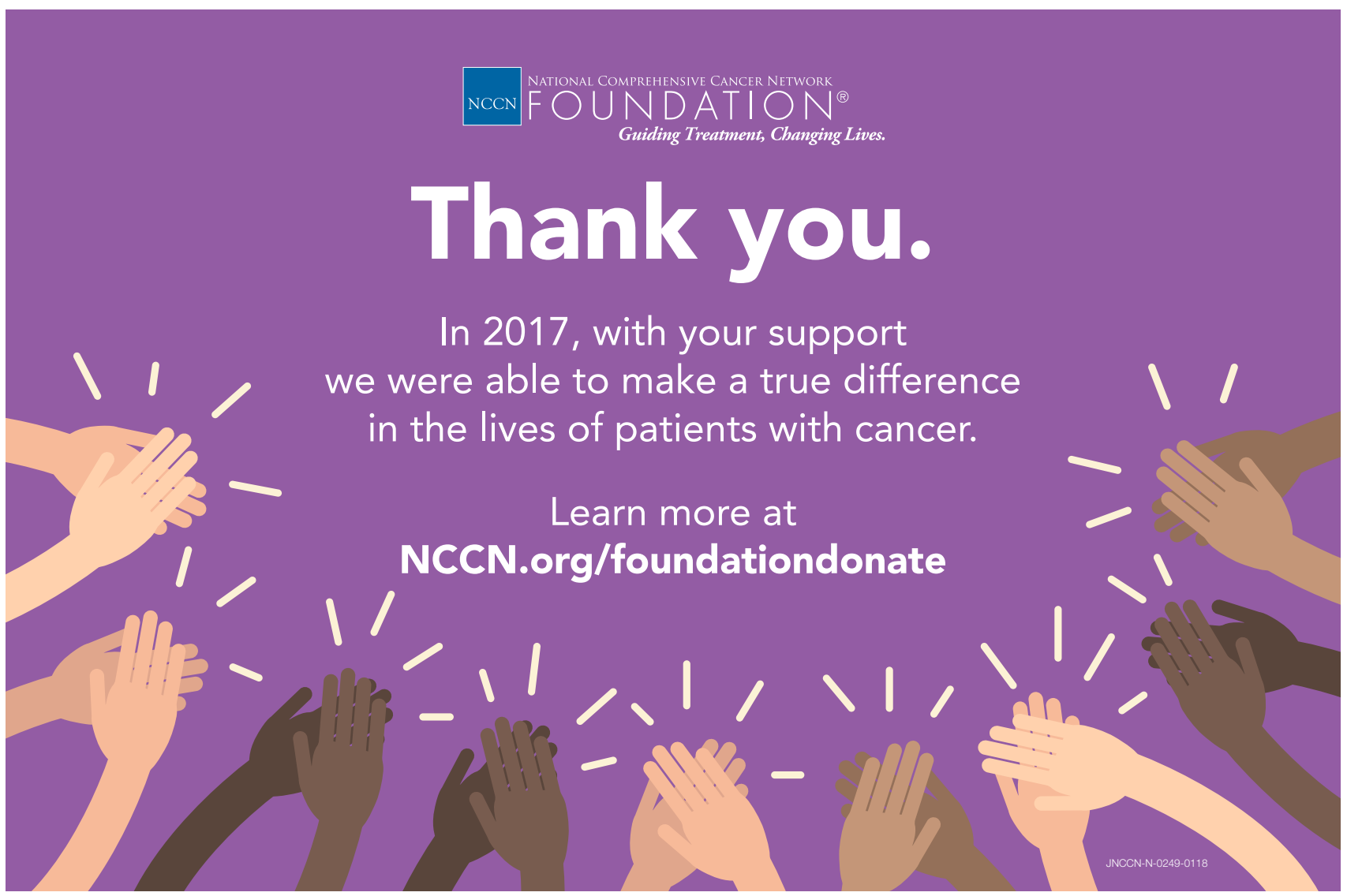

